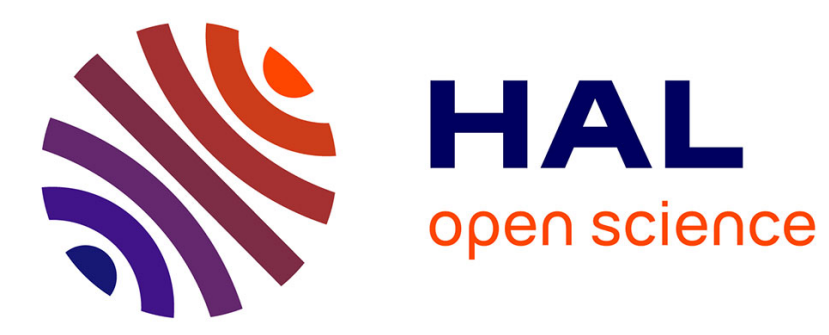

\title{
Water dimer equilibrium constant calculation: A quantum formulation including metastable states
}

Claude Leforestier

\section{To cite this version:}

Claude Leforestier. Water dimer equilibrium constant calculation: A quantum formulation including metastable states. Journal of Chemical Physics, 2014, 140, pp.074106. 10.1063/1.4865339 . hal00949455

\section{HAL Id: hal-00949455 \\ https://hal.science/hal-00949455}

Submitted on 19 Feb 2014

HAL is a multi-disciplinary open access archive for the deposit and dissemination of scientific research documents, whether they are published or not. The documents may come from teaching and research institutions in France or abroad, or from public or private research centers.
L'archive ouverte pluridisciplinaire HAL, est destinée au dépôt et à la diffusion de documents scientifiques de niveau recherche, publiés ou non, émanant des établissements d'enseignement et de recherche français ou étrangers, des laboratoires publics ou privés. 


\title{
Water dimer equilibrium constant calculation: A quantum formulation including metastable states
}

\author{
Claude Leforestier ${ }^{\mathrm{a})}$ \\ Institut Charles Gerhardt, CNRS 5253, CC 15.01, Université Montpellier II-CNRS, \\ 34095 Montpellier Cedex 05, France
}

(Received 28 November 2013; accepted 30 January 2014; published online 19 February 2014)

\begin{abstract}
We present a full quantum evaluation of the water second virial coefficient $B(T)$ based on the Takahashi-Imada second order approximation. As the associated trace $\operatorname{Tr}\left[e^{-\beta \mathbf{H}_{A B}}-e^{-\beta \mathbf{H}_{A B}^{o}}\right]$ is performed in the coordinate representation, it does also include contribution from the whole continuum, i.e., resonances and collision pairs of monomers. This approach is compared to a Path Integral Monte Carlo evaluation of this coefficient by Schenter [J. Chem. Phys. 117, 6573 (2002)] for the TIP4P potential and shown to give extremely close results in the low temperature range (250-450 K) reported. Using a recent $a b$ initio flexible potential for the water dimer, this new formulation leads to very good agreement with experimental values over the whole range of temperatures available. The virial coefficient is then used in the well known relation $K_{p}(T)=-\left(B(T)-b_{M}\right) / R T$ where the excluded volume $b_{M}$ is assimilated to the second virial coefficient of pure water monomer vapor and approximated from the inner repulsive part of the interaction potential. This definition, which renders $b_{M}$ temperature dependent, allows us to retrieve the $38 \mathrm{~cm}^{3} \mathrm{~mol}^{-1}$ value commonly used, at room temperature. The resulting values for $K_{p}(T)$ are in agreement with available experimental data obtained from infrared absorption spectra of water vapor. () 2014 AIP Publishing LLC. [http://dx.doi.org/10.1063/1.4865339]
\end{abstract}

\section{INTRODUCTION}

The importance of the water dimer $\left(\mathrm{H}_{2} \mathrm{O}\right)_{2}$ stems from its potential role in several atmospheric processes. It has been specifically invoked in the excess absorption of solar radiation, ${ }^{1-6}$ the water continuum absorption in the far infrared, ${ }^{7,8}$ homogeneous nucleation of water into droplets and ice, ${ }^{9,10}$ and catalysis of important chemical reactions, ${ }^{11,12}$ such as acid rain formation. For a long time however, its actual influence remained elusive as its concentration and variation with temperature were not clearly established. The only available values for the dimerization constant $K_{p}(T)$ were from the thermal conductivity of the classic steam experiments of Curtiss et al. ${ }^{13}$ which provided results over a very limited temperature range (358-386 K). Another source of experimental data came from the measurement of the second virial coefficient (SVC) $B(T)$ through the approximate relation, ${ }^{14}$ to be discussed later on,

$$
K_{p}(T) \simeq-\frac{1}{R T}\left(B(T)-b_{0}\right),
$$

where $b_{0}$ is the so-called excluded volume.

In the last decade, new experiments were specifically devoted to assess this problem. These studies have mainly concentrated on the infrared absorption in the fundamental, first overtone, and first combination band of the water monomer vibrations, ${ }^{15-19}$ but studies in the visible were also realized. Recently, Tretyakov et $a l^{20}$ directly measured the dimer absorption of millimeter-wave radiation in water vapor at $296 \mathrm{~K}$ and equilibrium conditions. From all these studies, one can es-

\footnotetext{
a)Electronic mail: claude.leforestier@univ-montp2.fr
}

timate a $K_{p}$ value lying between 0.03 and $0.06 \mathrm{~atm}^{-1}$ at room temperature.

So far, theoretical calculation of the dimer equilibrium constant relied on the explicit evaluation of its partition function ${ }^{21} Q_{D}(T)$

$$
K_{p}(T)=\frac{p_{D}}{p_{M}^{2}}=\frac{1}{k_{B} T} \frac{Q_{D}}{\lambda_{D}^{3}}\left(\frac{\lambda_{M}^{3}}{Q_{M}}\right)^{2} \times e^{D_{0} / k_{B} T},
$$

where $\lambda$ is the thermal de Broglie wavelength, or, equivalently, from the thermochemical relation ${ }^{22,23}$

$$
K_{p}(T)=\exp \left\{-\Delta G^{o} / R T\right\} / p^{o} .
$$

In most cases, the $Q_{D}$ partition function or the Gibbs free energy of formation $\Delta G^{o}$ were evaluated from frequencies calculated at the equilibrium geometry of the dimer and corrected for anharmonicity for the water $\operatorname{dimer}^{24-26}$ or the formic acid dimer. ${ }^{27}$ More recently, this function has been computed from an explicit calculation of the energy levels on an $a b$ initio six-dimensional energy surface, further adjusted to reproduce the Vibration-Rotation-Tunnelling (VRT) $\mathrm{THz}$ spectrum. ${ }^{28,29}$ This approach culminated in the explicit consideration of monomers' flexibility, ${ }^{30}$ as described by a 12-dimensional potential fitted to VRT experiments. ${ }^{31}$ In the temperature range of interest for atmospheric processes $(\sim 300 \mathrm{~K})$, such a calculation turned out to be very difficult due to the following reasons:

(i) convergence of the partition function requires consideration of very high $J$ values of the dimer, up to $J \sim 70$, which were handled by extrapolation, using the 
$J$-shifting approximation, ${ }^{32,33}$ from the results obtained for $(J \leq 5, K \leq 5)$ in the symmetric top approximation;

(ii) for most of these $(J, K)$ values, all vibrational levels up to dissociation are significantly populated, and were computed by means of the Lanczos algorithm;

(iii) consequently, resonant or metastable states are expected to play a role ${ }^{34,35}$ which was totally ignored in the calculation.

It should also be noted that the empirical flexible potential ${ }^{31}$ used in this study turned out to be too attractive $\left(D_{0} \simeq 1230 \mathrm{~cm}^{-1}\right)$ with respect to the recent experimental ${ }^{36}$ value $D_{0}=1105 \mathrm{~cm}^{-1}$.

The problem of the contribution of resonances to the SVC, which is related to $K_{p}(T)$ (see Eq. (1)), was addressed initially by Hirschfelder and co-workers ${ }^{14,37,38}$ who wrote its expression as comprising three different terms,

$$
B(T)=B_{f}(T)+B_{b}(T)+B_{m}(T),
$$

arising, respectively, from collision, bound, and metastable pairs of monomers. They were able to compute these different contributions, using classical collision theory, in the case of spherically symmetric interaction model potentials. For more complex potentials, they proposed to use the simple relation (Eq. (1)) where $b_{0}$ was identified to the free (unbound) contribution $B_{f}(T)$. This relation was used by Curtiss et al. ${ }^{13}$ with an empirical value $b_{0}=38.5 \mathrm{~cm}^{3} \mathrm{~mol}^{-1}$ to estimate the equilibrium constant of water.

The contribution of metastable and collision pairs to the virial coefficient was later formally addressed by $\mathrm{Smith}^{39,40}$ in a quantum collisional framework. The basic idea is to assume that pairs of molecules involved in any collision act like a single bound molecule for the duration of the collision lifetime, and the formulation relies on the collision lifetime matrix $\mathbf{Q}=i \hbar \mathbf{S}^{\dagger} d \mathbf{S} / d E$ which provides the complex energy spectrum. The dimer partition function $Z_{D}$ is then obtained from the expression

$$
Z_{D}=\int_{D_{0}}^{\infty} e^{-\beta E} \operatorname{Tr}[\mathbf{Q}(E)] d E .
$$

The calculation of the collision lifetime matrix $\mathbf{Q}$ for the much simpler case of the $\mathrm{HO}_{2}$ system ${ }^{41}$ already represented a tour de force. Such a procedure cannot be envisioned for the water dimer as this matrix would have to be determined at every resonance energy and for every relevant total angular momentum value.

Generalization of the classical phase space partition method of Stogryn and Hirschfelder to more realistic potentials was studied by Rainwater, ${ }^{42}$ Vigasin and coworkers, ${ }^{43-46}$ and by Schenter ${ }^{47}$ in a quantum mechanical formulation. Schenter et $a l .{ }^{48}$ thus showed that the equilibrium constant of water dimer can change by two orders of magnitude in the range $200-500 \mathrm{~K}$ depending on the choice of the partition.

In this work, we propose to determine the equilibrium constant from Eq. (1), where the SVC $B(T)$ is evaluated within a quantum quasi-exact formulation which does include its three different contributions as explicited in Eq. (4), and from a simple physical definition of the excluded volume $b_{0}(T)$ based on the equation of state of a monomer-dimer mixture. The outline of this paper is as follows. In Sec. II, we briefly recall the system definition used for a water dimer with flexible monomers, and present our quantum formulation of the SVC. Section III first elaborates on the relation (1) between this coefficient and the equilibrium constant, and uses it to determine it over a whole range of temperatures, which are compared to available experimental values. Finally, some conclusions are drawn in Sec. IV.

\section{SECOND VIRIAL COEFFICIENT}

In this section, we present quantum calculations of the SVC $B(T)$ of water to different approximation levels related to the way the density operator is handled. These results will be compared both to their available exact quantum, obtained by the Path Integral Monte Carlo (PIMC) method, and experimental counterparts. But first, we briefly recall the formulation used to handle two interacting, rigid or flexible, monomers as required to perform these calculations. This latter method has been described in full detail in a recent paper. ${ }^{49}$

\section{A. System description}

After reduction to its center of mass, an $\mathrm{AB}$ complex made of two rigid molecules $\mathrm{A}$ and $\mathrm{B}$ can be described in terms of the relative vector $\mathbf{R}$ and two sets of Euler angles $\boldsymbol{\Omega}^{A}$ and $\boldsymbol{\Omega}^{B}, \boldsymbol{\Omega}^{X}$ standing for the three angles labelled $\left(\chi^{X}, \theta^{X}\right.$, $\left.\varphi^{X}\right)$. We use the rigid monomers Body-Fixed formulation of Brocks et al. ${ }^{50}$

$$
\begin{aligned}
\hat{H}_{A B}^{(B F)}= & -\frac{\hbar^{2}}{2 \mu_{A B}} \frac{1}{R} \frac{\partial^{2}}{\partial R^{2}} R+\hat{H}_{r o t}^{A}+\hat{H}_{r o t}^{B}+V_{A B}\left(R, \boldsymbol{\Omega}^{A}, \boldsymbol{\Omega}^{B}\right) \\
& +\frac{1}{2 \mu_{A B} R^{2}}\left\{\hat{\boldsymbol{J}}^{2}+\hat{\boldsymbol{J}}_{A B}^{2}-2 \hat{\boldsymbol{J}}_{A B} . \hat{\boldsymbol{J}}\right\},
\end{aligned}
$$

where $\mu_{A B}$ is the reduced mass, $\hat{H}_{r o t}^{X}$ and $\hat{\boldsymbol{j}}_{X}$ are, respectively, the rotational Hamiltonian and angular momentum of monomer $X, \hat{\boldsymbol{j}}_{A B}=\hat{\boldsymbol{j}}_{A}+\hat{\boldsymbol{j}}_{B}$ is the coupled internal rotational angular momentum, and $\hat{\boldsymbol{J}}=\hat{\boldsymbol{J}}_{A B}+\hat{\boldsymbol{L}}$ is the total angular momentum $(\hat{\boldsymbol{L}}$ is the relative angular momentum between the monomers' centers of mass).

The kinetic energy operator (KEO) of Eq. (6) leads to simple matrix elements in the overall spectral basis set

$$
\begin{aligned}
\mathcal{B}= & \{|n\rangle\} \otimes\left\{\left|j_{A}, k_{A}, \omega_{A}\right\rangle\right\} \otimes\left\{\left|j_{B}, k_{B}, \omega_{B}\right\rangle\right\} \otimes\{|J, K, M\rangle\} \\
& \left(\omega_{A}+\omega_{B}=K\right),
\end{aligned}
$$

where $\{|n\rangle\}$ is an appropriate basis for the interfragment distance $R,|j, k, \omega\rangle$ is a Wigner function, and $\{|J, K, M\rangle\}$ is the Wigner basis set associated with the overall rotation of the complex. The basis $\mathcal{B}$ can be projected onto the different Irreducible Representations (Irreps) $\Gamma$ of the molecular symmetry group ( $G_{16}$ for water dimer) governing the system ${ }^{51}$

$$
\mathcal{B}=\bigoplus_{\Gamma}\{|n\rangle \otimes|v ; \Gamma\rangle\}
$$

The most compact representation for a rigid potential energy $V_{A B}\left(R, \boldsymbol{\Omega}^{A}, \boldsymbol{\Omega}^{B}\right)$ is the six-dimensional grid $\left\{\varphi_{g} \times \chi_{q}^{A}\right.$ 
$\left.\times \chi_{s}^{B} \times \theta_{\alpha}^{A} \times \theta_{\beta}^{B} \times R_{p}\right\}$, where $\varphi=\varphi^{A}-\varphi^{B}$. Actually, the intermolecular potential $V_{A B}$ does not depend on $\Phi=\varphi^{A}$ $+\varphi^{B}$, and this angle serves as the third angle orientating the Body Fixed frame. ${ }^{50}$ Transformations between the spectral and grid representations, as required to act the potential operator on a wavefunction, are performed by means of a sixdimensional pseudospectral scheme described previously. ${ }^{52}$ This pseudospectral scheme, which relies on gaussian quadratures and fast Fourier transforms, allows one to easily compute any integral of the form

$$
\int \ldots \int d R d \boldsymbol{\Omega}^{A} d \mathbf{\Omega}^{B} \hat{O} F\left(R, \boldsymbol{\Omega}^{A}, \boldsymbol{\Omega}^{B}\right),
$$

where $\hat{O}$ is some operator. The method consists in first expressing the function $F\left(R, \boldsymbol{\Omega}^{A}, \boldsymbol{\Omega}^{B}\right)$, defined by its value on the grid, in terms of the $\mathcal{B}$ basis set functions by means of the inverse transform of the pseudospectral scheme. One can then apply the operator $\hat{O}$ in the basis set, transform back to the grid representation, and evaluate the integral by the underlying quadratures. In practice, one first performs integration over the Euler angles $\boldsymbol{\Omega}^{A}$ and $\boldsymbol{\Omega}^{B}$. The resulting $\mathcal{F}(R)$ function is finally integrated by means of a Simpson quadrature.

\section{B. Flexibility correction}

The principle of flexible calculations has been described in full detail in a recent paper $^{49}$ which also introduced the flexible CCpol-8sf potential to be used in this work. We only recall here its main features for sake of clarity. In order to deal with the flexibility of the monomers, we replace the above rigid potential $V_{A B}\left(R, \boldsymbol{\Omega}^{A}, \boldsymbol{\Omega}^{B}\right)$ by its adiabatic counterpart $V_{A B}^{a d}\left(R, \boldsymbol{\Omega}^{A}, \boldsymbol{\Omega}^{B}\right)$ which takes into account the variation of the intramolecular zero-point energy as a function of the intermolecular geometry. Such an adiabatic approximation is justified by the high frequencies of these modes $(1595,3657$, and $3756 \mathrm{~cm}^{-1}$ ) as compared to dissociation energy $D_{0} \simeq 1105 \mathrm{~cm}^{-1} .{ }^{36}$ More specifically, if we denote, respectively, by $\mathbf{Q}$ and $\mathbf{q}^{X}$ the intermolecular coordinates and the intramolecular ones of monomer $\mathrm{X}$, the flexible, 12dimensional, potential is expanded as

$$
\begin{aligned}
V_{A B}\left(\mathbf{q}^{A}, \mathbf{q}^{B}, \mathbf{Q}\right)= & V_{\mathrm{opt}}(\mathbf{Q})+V^{A}\left(\mathbf{q}^{A} ; \mathbf{Q}\right)+V^{B}\left(\mathbf{q}^{B} ; \mathbf{Q}\right) \\
& +\Delta V_{A B}\left(\mathbf{q}^{A}, \mathbf{q}^{B}, \mathbf{Q}\right)
\end{aligned}
$$

where $V_{\text {opt }}(\mathbf{Q})$ refers to the intermolecular potential at optimized intramolecular geometries $\left(\mathbf{q}_{\mathrm{opt}}^{A}, \mathbf{q}_{\mathrm{opt}}^{B}\right)$ for the geometry $\mathbf{Q}$,

$$
\left.\frac{\partial V\left(\mathbf{q}^{A}, \mathbf{q}^{B}, \mathbf{Q}\right)}{\partial \mathbf{q}^{X}}\right|_{\mathbf{q}_{\mathrm{opt}}^{X}}=\mathbf{0} \quad(X=A, B),
$$

$V^{X}\left(\mathbf{q}^{X} ; \mathbf{Q}\right)$ corresponds to single-monomer correction, e.g.,

$$
V^{A}\left(\mathbf{q}^{A} ; \mathbf{Q}\right)=V\left(\mathbf{q}^{A}, \mathbf{q}_{\mathrm{opt}}^{B} ; \mathbf{Q}\right)-V_{\mathrm{opt}}(\mathbf{Q}),
$$

and $\Delta V_{A B}\left(\mathbf{q}^{A}, \mathbf{q}^{B}, \mathbf{Q}\right)$ is the residual potential coupling term.

At each point of the six-dimensional $\mathbf{Q}$-grid, the ground adiabatic potential $V_{A B}^{a d}(\mathbf{Q})$ can be solved either within the local monomer model approximation, ${ }^{31,53,54}$ i.e., neglecting the residual term $\Delta V_{A B}\left(\mathbf{q}^{A}, \mathbf{q}^{B}, \mathbf{Q}\right)$, or in a full variational ap- proach explicitly considering it. We used here this later approach, more computationally demanding as it corresponds to solving a six-dimensional vibrational problem at each point of the six-dimensional Q-grid. However, it should be noted that the local monomer approximation actually leads to negligible changes (less than $0.1 \%$ ) in the computed SVC values for all temperatures considered here.

Another consequence of flexibility is to render the monomer rotational constants $\Gamma_{x x}, \Gamma_{y y}$, and $\Gamma_{z z}$ (entering the definition of the $\hat{H}_{r o t}^{A}$ and $\hat{H}_{r o t}^{B}$ operators) dependent on the $\mathbf{Q}$ intermolecular geometry,

$$
\hat{H}_{r o t}^{X}=\frac{1}{2} \hat{\boldsymbol{\jmath}}_{X}^{\dagger}\left(\begin{array}{ccc}
\Gamma_{x x}^{X}(\mathbf{Q}) & 0 & 0 \\
0 & \Gamma_{y y}^{X}(\mathbf{Q}) & 0 \\
0 & 0 & \Gamma_{z z}^{X}(\mathbf{Q})
\end{array}\right) \hat{\boldsymbol{J}}_{X},
$$

where the $\Gamma_{\alpha \alpha}^{X}(\mathbf{Q})$ rotational constants are averaged over the instantaneous adiabatic monomer states. Such a dependence was investigated in our paper, and was shown to be essentially accounted for by only retaining the $R$-dependence, provided that one defines effective rotational quantities $\widetilde{\Gamma}_{z z}^{X}(R)$ obtained from averaging over the $\boldsymbol{\Omega}^{A}, \boldsymbol{\Omega}^{B}$ Euler angles. Typically, such an approximation allowed us to retrieve exact energies within less than $0.1 \%$ up to more than half the dissociation energy.

\section{Different approximations}

The standard definition of the $\mathrm{SVC}^{21}$ reads

$$
B(T)=V\left(\frac{1}{2}-\frac{Q_{d}}{Q_{m}^{2}}\right),
$$

where $Q_{m}$ and $Q_{d}$ are the monomer and dimer partition functions, respectively, and $V$ is the volume of the vessel containing the gas. As we are considering here flexible monomers, $Q_{m}$ and $Q_{d}$ should in principle receive contribution from the intramolecular modes of each monomer. However, the high frequencies of these modes render such a contribution negligible in the temperature range $200-1000 \mathrm{~K}$ considered here (e.g., $\left.Q_{1, v i b}(800 \mathrm{~K}) \simeq 1.06\right)$. We will thus ignore the effect of excited intramolecular states, both in $Q_{m}$ and $Q_{d}$, leading to

$$
Q_{m}=Q_{m, t r} \times Q_{m, r o t} .
$$

It should be kept in mind that the change in zero-point energy of these modes within the dimer is accounted for in the definition of the adiabatic potential $V_{A B}^{a d}(\mathbf{Q})$. The SVC formulation for flexible monomers can thus be recast into the standard rigid monomer one, provided that the intermolecular potential $V_{A B}(\mathbf{Q})$ entering Eq. (6) be replaced by its adiabatic counterpart $V_{A B}^{a d}(\mathbf{Q})$, and the $\mathbf{Q}$-dependence of rotational constants (Eq. (11)) be considered.

We now capitalize on the rigid monomer second virial formulation of Wormer, ${ }^{55}$ recalling the essential steps and the changes we bring in his derivation. The SVC can be written in terms of the density operator

$$
B(T)=\frac{V}{2 Q_{m}^{2}} \operatorname{Tr}\left[e^{-\beta \hat{\mathcal{H}}_{A B}^{o}}-e^{-\beta \hat{\mathcal{H}}_{A B}}\right]_{S F},
$$

where $\hat{\mathcal{H}}_{A B}^{o}=\hat{\mathcal{H}}_{A}^{o}+\hat{\mathcal{H}}_{B}^{o}$ stands for the space-fixed total hamiltonian of the two non-interacting monomers, $\hat{\mathcal{H}}_{A B}$ takes 
into account their interaction potential $V_{A B}$ (or $V_{A B}^{a d}$ ), and SF recalls that the trace has to be evaluated in the space-fixed frame, that is considering the six coordinates $\vec{r}_{X}$ (position in space) and $\boldsymbol{\Omega}^{X}$ (orientation) of each monomer.

We will consider different approximations to the $e^{-\beta \hat{\mathcal{H}}_{A B}}$ density operator of the form

$$
e^{-\beta \hat{\mathcal{H}}_{A B}} \simeq e^{-\beta \hat{\mathcal{H}}_{A B}^{o}} \times e^{-\beta V_{A B}} \times \mathcal{F}
$$

where $\mathcal{F}$ is a function, not an operator, to be discussed later on. Our formulation departs now from Wormer's one as he relied instead on the following expansion:

$$
e^{-\lambda \beta \hat{\mathcal{H}}_{A B}}=e^{-\lambda \beta V_{A B}} \hat{T}(\lambda) e^{-\lambda \beta \hat{\mathcal{H}}_{A B}^{o}},
$$

and searched for an iterative solution of $\hat{T}(\lambda)$.

This crucial approximation (Eq. (15)) allows one to explicit the coefficient, using integration over physical space to evaluate the trace

$$
\begin{aligned}
B(T)= & \frac{V}{2 Q_{m}^{2}} \int \ldots \int d \mathbf{x}_{A} d \mathbf{x}_{B}\left\langle\mathbf{x}_{A} \mathbf{x}_{B}\left|e^{-\beta \hat{\mathcal{H}}_{A B}^{o}}\right| \mathbf{x}_{A} \mathbf{x}_{B}\right\rangle \\
& \times\left\{1-e^{-\beta V_{A B}\left(\mathbf{x}_{A}, \mathbf{x}_{B}\right)} \mathcal{F}\left(\mathbf{x}_{A} \mathbf{x}_{B}\right)\right\},
\end{aligned}
$$

where $\mathbf{x}_{X}$ stands for the six coordinates of monomer $X$, and we have made use of the local character of $V_{A B}$ and $\mathcal{F}$. Using the independence of the Slater sum $\left\langle\mathbf{x}_{X}\left|e^{-\beta \hat{\mathcal{H}}_{X}^{o}}\right| \mathbf{x}_{X}\right\rangle$ over $\mathbf{x}_{X}$,

$$
\left\langle\mathbf{x}_{X}\left|e^{-\beta \hat{\mathcal{H}}_{X}^{o}}\right| \mathbf{x}_{X}\right\rangle=\frac{Q_{m, t r}}{V} \times \frac{Q_{m, r o t}}{8 \pi^{2}},
$$

the coefficient expression simplifies to

$$
B(T)=\frac{1}{2 V\left(8 \pi^{2}\right)^{2}} \int \ldots \int d \mathbf{x}_{A} d \mathbf{x}_{B}\left\{1-e^{-\beta V_{A B}\left(\mathbf{x}_{A}, \mathbf{x}_{B}\right)} \mathcal{F}\left(\mathbf{x}_{A} \mathbf{x}_{B}\right)\right\} .
$$

Finally, noting that $V_{A B}$ and $\mathcal{F}$ do not depend on the position of the center of mass (c.m.) of the dimer, one can perform the c.m. reduction and integrate its coordinates over the vessel volume $V$

$$
\begin{aligned}
B(T)= & \frac{1}{128 \pi^{4}} \int \ldots \int\left\{1-e^{-\beta V_{A B}\left(R, \boldsymbol{\Omega}^{A}, \boldsymbol{\Omega}^{B}\right)} \mathcal{F}\left(\mathbf{R}, \boldsymbol{\Omega}^{A}, \boldsymbol{\Omega}^{B}\right)\right\} \\
& \times d \mathbf{R} d \boldsymbol{\Omega}^{A} d \mathbf{\Omega}^{B} .
\end{aligned}
$$

The above integral is more easily evaluated after transformation to the body-fixed frame introduced in Sec. II A, which we will consider from now on.

\section{Classical approximation}

The well known classical approximation ${ }^{56}$ consists here in using $\mathcal{F}=1$, leading to

$$
B_{c l}(T)=\frac{1}{128 \pi^{4}} \int \ldots \int\left\{1-e^{-\beta V_{A B}\left(R, \boldsymbol{\Omega}^{A}, \boldsymbol{\Omega}^{B}\right)}\right\} d \mathbf{R} d \boldsymbol{\Omega}^{A} d \boldsymbol{\Omega}^{B} .
$$

\section{Takahashi and Imada second-order approximation}

A second order approximation has been proposed by Takahashi and Imada ${ }^{57}$ (TI) in terms of the double commu- tator

$$
\operatorname{Tr}\left[e^{-\beta \hat{H}_{A B}}\right] \simeq \operatorname{Tr}\left[e^{-\beta \hat{H}_{A B}^{o}} e^{-\beta\left(V_{A B}+\frac{\beta^{2}}{24}\left[V_{A B},\left[\hat{H}_{A B}^{o}, V_{A B}\right]\right]\right)}\right],
$$

where $\hat{H}_{A B}^{o}$ means the body-fixed counterpart of $\hat{\mathcal{H}}_{A B}^{o}$ and corresponds to the operator of Eq. (6) minus the potential. Within this approximation, the $\mathcal{F}$ quantity introduced in Eq. (15) corresponds to

$$
\mathcal{F}=e^{-\frac{\beta^{3}}{24}\left[V_{A B},\left[\hat{H}_{A B}^{o}, V_{A B}\right]\right]}
$$

and will be shown later to actually be a function despite its operatorial expression.

Following Schenter, ${ }^{58}$ the contribution of this double commutator is usually taken into account through classical statistical simulations: it consists in adding the term

$$
+\frac{\hbar^{2} \beta^{2}}{24} \sum_{i=1}^{2}\left[\frac{\mathbf{F}_{i}^{2}}{M}+\sum_{\alpha=1}^{3} \frac{\tau_{\alpha i}}{I_{\alpha}}\right]
$$

to the $V_{A B}$ potential in the second virial classical expression (Eq. (20)). In the above equation, $\mathbf{F}_{i}$ is the force on molecule $i$ exerted by the partner, the $\tau$ 's are the components of the torque on the molecule, $M$ is the mass of each molecule, and the I's are the principal moments of inertia. Calculations are then carried out by Monte Carlo sampling over the Euler angles, and a numerical quadrature for the $R$ distance. ${ }^{59}$

In the work presented here, we consider instead an exact quantum mechanical evaluation of the double commutator of Eq. (21). To this aim, we first explicit $\hat{H}_{A B}^{o}$ (rigid case) in the form $^{55}$

$$
\begin{aligned}
\hat{H}_{A B}^{o}= & \frac{\hat{P}_{R}^{2}}{2 \mu_{A B}}+\frac{1}{2} \sum_{\alpha X} \Gamma_{\alpha \alpha}^{X} \hat{J}_{\alpha X}^{2} \\
& +\frac{1}{2 \mu_{A B} R^{2}}\left\{\hat{\boldsymbol{J}}^{2}+\hat{\boldsymbol{J}}_{A B}^{2}-2 \hat{\boldsymbol{J}}_{A B} . \hat{\boldsymbol{J}}\right\},
\end{aligned}
$$

where $\hat{P}_{R}=-i \hbar R^{-1}(\partial / \partial R) R$. Straightforward algebra allows one to show the following relations:

$$
\begin{aligned}
& {\left[V_{A B},\left[\hat{P}_{R}^{2}, V_{A B}\right]\right]=2 \hbar^{2}\left(\frac{\partial V_{A B}}{\partial R}\right)^{2},} \\
& {\left[V_{A B},\left[\hat{\jmath}_{\alpha X}^{2}, V_{A B}\right]\right]=-2\left(\hat{\jmath}_{\alpha X} V_{A B}\right)^{2} .}
\end{aligned}
$$

The $\hat{\boldsymbol{J}}^{2}$ and $\hat{\boldsymbol{J}}_{A B} . \hat{\boldsymbol{J}}$ operators contribute for zero as they give rise to null $\hat{J}_{\alpha} V_{A B}$ terms because the potential is invariant in the overall rotation of the dimer. But, as stressed by $\mathrm{T}$ Pack, ${ }^{56}$ the $\hat{\boldsymbol{j}}_{A B}^{2}$ terms do contribute and can be evaluated from Eq. (26).

One finally obtains for the double commutator expression

$$
\begin{aligned}
{\left[V_{A B},\left[\hat{H}_{A B}^{o}, V_{A B}\right]\right]=} & \frac{\hbar^{2}}{\mu_{A B}}\left(\frac{\partial V_{A B}}{\partial R}\right)^{2}-\sum_{\alpha X} \Gamma_{\alpha \alpha}^{X}\left(\hat{\jmath}_{\alpha X} V_{A B}\right)^{2} \\
& -\frac{1}{\mu_{A B} R^{2}} \sum_{\alpha}\left(\hat{\jmath}_{\alpha A} V_{A B}+\hat{\jmath}_{\alpha B} V_{A B}\right)^{2}
\end{aligned}
$$

It should be noted that, because the angular momentum operators are hermitian $\hat{\jmath}_{\alpha}=-\imath \hbar \partial / \partial \ldots$, the last two terms in the above expression are positive. 
If performing flexible calculations, the above TI correction has to be modified in two ways. The first, trivial, one is to replace the $V_{A B}$ potential by its adiabatic version $V_{A B}^{a d}$ as defined in Sec. II B. A second modification concerns the middle term of Eq. (27). This term was established for rotational operators $\hat{H}_{\text {rot }}^{X}$ displaying fixed rotational constants. It was mentioned in Sec. II B that a consequence of flexibility was to turn these constants Q-dependent. However, considering $R$-dependent effective quantities $\widetilde{\Gamma}_{z z}^{X}(R)$, obtained from averaging, allows one to retrieve quasi-exact energies. The corresponding rotational operators thus display the modified expression

$$
\hat{H}_{r o t}^{X}=\sum_{\alpha} \widetilde{\Gamma}_{\alpha \alpha}^{X}(R) \hat{\jmath}_{\alpha X}^{2}
$$

as $\hat{J}_{\alpha X}$ does not contain any $R$-derivative. The modified TI correction Eq. (27) corresponding to flexible monomers displays the following expression:

$$
\begin{aligned}
{\left[V_{A B}^{a d},\left[\hat{H}_{A B}^{o}, V_{A B}^{a d}\right]\right]=} & \frac{\hbar^{2}}{\mu_{A B}}\left(\frac{\partial V_{A B}^{a d}}{\partial R}\right)^{2}-2 \sum_{\alpha X} \widetilde{\Gamma}_{\alpha \alpha}^{X}\left(\hat{\jmath}_{\alpha X} V_{A B}^{a d}\right)^{2} \\
& -\frac{1}{\mu_{A B} R^{2}} \sum_{\alpha}\left(\hat{\jmath}_{\alpha A} V_{A B}^{a d}+\hat{\jmath}_{\alpha B} V_{A B}^{a d}\right)^{2} .
\end{aligned}
$$

It is customary to define the effective potential $\widetilde{\mathbf{V}}_{A B}$ from

$$
\widetilde{V}_{A B}=V_{A B}+\frac{\beta^{2}}{24}\left[V_{A B},\left[\hat{H}_{A B}^{o}, V_{A B}\right]\right]
$$

or from the correction Eq. (29) in case of flexible monomers, which recasts the Takahashi-Imada approximation in a form similar to the classical one (Eq. (20)),

$$
B_{t i}(T)=\frac{1}{128 \pi^{4}} \int \ldots \int\left\{1-e^{-\beta \widetilde{V}_{A B}\left(R, \boldsymbol{\Omega}^{A}, \boldsymbol{\Omega}^{B}\right)}\right\} d \mathbf{R} d \mathbf{\Omega}^{A} d \mathbf{\Omega}^{B} .
$$

We report in Table I the $B(T)$ values as obtained from the above quantum TI and classical approximations for the rigid TIP4P potential. ${ }^{60}$ Using this potential allowed us to compare with the results obtained by Schenter, ${ }^{58}$ respectively, from a quantum statistical (Path Integral Monte Carlo) method, the classical TI (Eq. (23)), and the standard classical approximations. One can note that the quantum TI and PIMC results agree within $1 \%$, except for the lowest temperature $(250 \mathrm{~K})$ where the quantum TI value lays halfway between the PIMC and classical TI ones. Calculation of $B(T)$ at any temperature is extremely fast, once the corrective term $\left[V_{A B},\left[\hat{H}_{A B}^{o}, V_{A B}\right]\right]$ in Eq. (30) has been computed: typical times are $26 \mathrm{~s}$ and $105 \mathrm{~s}$, respectively, on a 16-core processor.

\section{Results}

All the calculations presented in this work have been performed with the following basis specifications: (i) a Wigner basis set up to $j=11$ on each monomer, (ii) a radial basis set of 125 sine functions spanning the box $4 \leq R \leq 46 a_{o}$, using the same number of grid points. Fig. 1 displays the SVC $B(T)$ in the range $250-1000 \mathrm{~K}$ as obtained within the TI and classical approximations using the recently developed flexible
TABLE I. Second virial coefficient $B(T)$ values $\left(\mathrm{cm}^{3} \mathrm{~mol}^{-1}\right)$, using the TIP4P potential. Schenter's results ${ }^{58}$ correspond, respectively, to a quantum statistical method (Path Integral Monte Carlo), a classical statistical formulation of the Takahashi-Imada approximation and the classical approximation. Present results were obtained, respectively, from a quantum formulation of the TI approximation, and in the classical approximation.

\begin{tabular}{ccccccc}
\hline \hline & \multicolumn{3}{c}{ Schenter } & & \multicolumn{2}{c}{ This work } \\
\cline { 2 - 4 } \cline { 6 - 7 }$T(\mathrm{~K})$ & PIMC & $\mathrm{TI}_{c l}$ & Clas. & & $\mathrm{TI}_{q u}$ & Clas. \\
\hline 200.0 & $\ldots$ & -72831 & -168650 & & -68766 & -168827 \\
250.0 & -8412 & -8877 & -15203 & & -8633 & -15213 \\
273.2 & -4450 & -4539 & -7065 & & -4452 & -7079 \\
300.0 & -2369 & -2439 & -3486 & & -2402 & -3486 \\
323.2 & -1557 & -1573 & -2122 & & -1553 & -2122 \\
373.2 & -752 & -756 & -936 & & -750 & -936 \\
400.0 & -552 & -555 & -666 & & -551 & -665 \\
423.2 & -434 & -439 & -515 & & -436 & -515 \\
448.2 & -338 & -350 & -404 & & -348 & -404 \\
473.2 & -279 & -286 & -325 & & -285 & -325 \\
\hline \hline
\end{tabular}

CCpol-8sf potential. ${ }^{49}$ We also report this coefficient obtained from the TI approximation but for the rigid version ${ }^{61}$ of this potential. In Figure 1 are also depicted the experimental values of Osborne et al. ${ }^{62}$ Eubank et al. ${ }^{63}$ and Kell et al.,${ }^{64}$ as well as their fit by Harvey and Lemmon. ${ }^{65}$ A detailed comparison at temperatures used in the experiments is given in Table II.

One can note a very good agreement between the experimentally fitted curve and the results obtained within the TI approximation using the flexible potential over the whole range of temperatures reported. As our evaluation of $B(T)$ is nearly exact, this agreement assesses the very high quality of the global CCpol-8sf potential, which was previously tested only on properties ${ }^{49}$ (VRT spectrum and IR shifts) mainly sampling the inter-monomer equilibrium distance. It also demonstrates that flexibility is required in the calculation of the SVC. Although the rigid curve is roughly left-translated by $8 \mathrm{~K}$ only

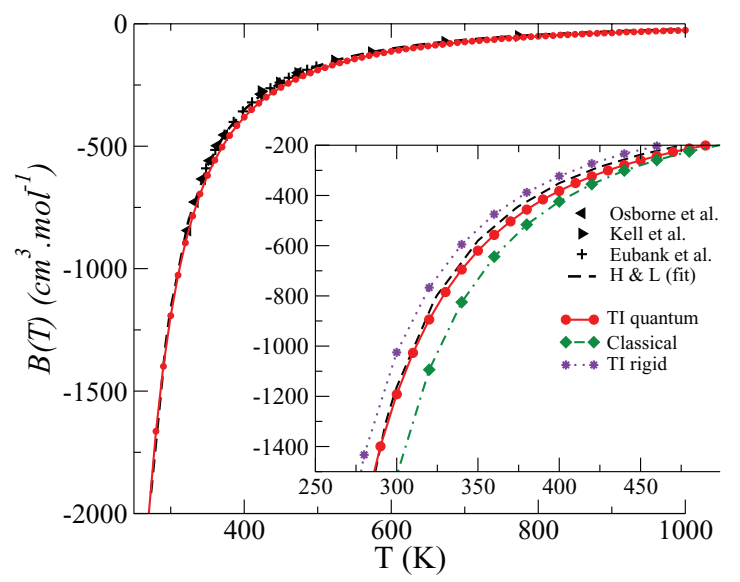

FIG. 1. Comparison of the calculated SVC $B(T)$ within the Takahashi-Imada quantum formulation (•) with experimental values: Ref. $62(\bullet)$, Ref. $64(\triangleright)$, Ref. 63 (+), fit (-) by Harvey and Lemmon in Ref. 65. The inset displays these same values in the $250-500 \mathrm{~K}$ range, as well as those obtained from different approximations: classical $(\bullet)$ and Takahashi-Imada rigid calculations $(*)$. 
TABLE II. Comparison of experimental second virial coefficient $B(T)$ values $\left(\mathrm{cm}^{3} \mathrm{~mol}^{-1}\right)$ obtained in earlier work to those calculated in this work using the quantum TI formulation.

\begin{tabular}{llllll}
\hline$T(\mathrm{~K})$ & Exp. $^{\mathrm{a}}$ & Exp. $^{\mathrm{b}}$ & Exp. $^{\mathrm{c}}$ & Fit $^{\mathrm{d}}$ & Calc. \\
\hline 323.13 & -844 & & & -817 & -859 \\
333.13 & -728 & & & -714 & -756 \\
343.13 & -634 & & & -629 & -671 \\
348.15 & & & -590 & -593 & -634 \\
353.13 & -559 & & & -560 & -600 \\
360.65 & & & -515 & -515 & -554 \\
363.13 & -498 & & & -501 & -540 \\
373.15 & -454 & & -453 & -452 & -488 \\
385.65 & & & -401 & -400 & -434 \\
398.15 & & & -358 & -357 & -388 \\
410.65 & & & -321 & -321 & -350 \\
423.15 & -287 & -275 & -290 & -290 & -317 \\
448.15 & & -240 & -240 & -241 & -264 \\
460.65 & & & -220 & -221 & -242 \\
473.15 & -200 & -201 & -203 & -203 & -223 \\
485.65 & & & -188 & -188 & -206 \\
498.15 & & & -174 & -174 & -191 \\
523.15 & & -150 & & -150 & -166 \\
573.15 & & -116 & & -116 & -128 \\
673.15 & & -74 & & -74 & -83 \\
773.15 & & -50 & & -50 & -57 \\
\hline \hline
\end{tabular}

${ }^{\mathrm{a}}$ From Osborne et al. ${ }^{62}$

${ }^{\mathrm{b}}$ From Eubank et al. ${ }^{63}$

${ }^{\mathrm{c}}$ From Kell et al. ${ }^{64}$

${ }^{\mathrm{d}}$ From their fit by Harvey and Lemmon. ${ }^{65}$

with respect to the flexible one, their quasi-vertical asymptotic behavior in the low temperature region leads to a $13 \%$ error at $300 \mathrm{~K}$. This contrasts with the recent claim by Donchev et $a l .{ }^{66}$ who reported a reduction of $20 \%-40 \%$ upon consideration of flexibility in this temperature range. Eventually, the rigid TI results finally converge to the flexible ones for high temperatures. This can be understood as the rigid potential uses the vibrationally averaged (free) monomer geometry which becomes preponderant at large separations, more heavily sampled at high temperatures. Comparison with the classical curve shows that the quantum correction is needed up to high temperatures. For example, the error ranges from $30 \%$ at $300 \mathrm{~K}$ to $10 \%$ at $500 \mathrm{~K}$.

\section{EQUILIBRIUM CONSTANT $K_{p}(T)$}

The integration method used to evaluate the SVC (Eq. (19)) does include the different contributions $B_{f}, B_{b}$, and $B_{m}$ as it corresponds to the trace of the integrand $\left(1-e^{-\beta \widetilde{V}_{A B}\left(R, \boldsymbol{\Omega}^{A}, \boldsymbol{\Omega}^{B}\right)}\right)$ expressed in the physical space. It is thus strictly equivalent to the trace performed in the energy representation

$$
\begin{aligned}
B(T)= & \sum_{\mathbf{b}}^{\text {bound }}\left\langle E_{\mathbf{b}}|(1-\ldots)| E_{\mathbf{b}}\right\rangle \\
& +\sum_{\mathbf{n}}^{\text {channels }} \int_{0}^{\infty} d E\left\langle E_{\mathbf{n}}|(1-\ldots)| E_{\mathbf{n}}\right\rangle
\end{aligned}
$$

where $\left\{\left|E_{\mathbf{b}}\right\rangle\right\}$ denotes the bound states $(E<0)$, and $\left\{\left|E_{\mathbf{n}}\right\rangle\right\}$ corresponds to the collision eigenstates $(E>0)$, which encompass the so-called free and metastable states.

\section{A. Relation between $B(T)$ and $K_{p}(T)$}

One considers the dimerization reaction

$$
2 M \leftrightarrow D .
$$

If both $\mathrm{M}$ and $\mathrm{D}$ are considered as ideal gases

$$
K_{p}=\frac{p_{D}}{p_{M}^{2}}=\frac{\alpha\left(1-\frac{\alpha}{2}\right)}{2(1-\alpha)^{2}} \frac{1}{p},
$$

where $\alpha$ is the dimerization yield and $p$ is the pressure at equilibrium, leading to the number of dimer moles,

$$
n_{D}=\frac{\alpha}{2} n_{0}=p K_{p} \frac{(1-\alpha)^{2}}{1-\frac{\alpha}{2}} n_{0},
$$

$n_{0}=n_{M}+2 n_{D}$ being the total number of moles of $\mathrm{M}$, either as monomers or within dimers.

The equation of state for the mixture reads

$$
\begin{aligned}
p V & =n_{M} R T+n_{D} R T=n_{0} R T-n_{D} R T \\
& =n_{0} R T+n_{0} p \times \overbrace{\left[-K_{p} R T \frac{(1-\alpha)^{2}}{1-\frac{\alpha}{2}}\right]}^{\text {observed } B(T)} .
\end{aligned}
$$

The above equation departs from the usual formulation for ideal mixtures (see, e.g., Lambert et al. ${ }^{67}$ ) in that we explicitly consider the dimerization yield $\alpha$ to be finite instead of taking the $\alpha \rightarrow 0$ limit. The way to take into account this nonzero value will be discussed later after we consider below the nonideality of the monomer gas. In such a case, the $p_{M}$ expression has to be modified according to

$$
p_{M} V=n_{M} R T+n_{M} b_{M} p_{M},
$$

where $b_{M}$ would be the SVC of $\mathrm{M}$ in the absence of any dimer in the mixture, and the definition of which is deferred to Sec. III B.

Equation (35) has thus to be corrected according to this contribution,

$$
n_{M} b_{M} p_{M} \simeq n_{0} p b_{M} \frac{(1-\alpha)^{2}}{1-\frac{\alpha}{2}}
$$

where we have made the approximation $p_{M} / p$ $\simeq(1-\alpha) /\left(1-\frac{\alpha}{2}\right)$ as $b_{M}$ represents a small contribution as compared to $B(T)$, and leads to the final expression for the equilibrium constant $K_{p}(T)$,

$$
K_{p}(T)=-\frac{1}{R T}\left[B(T) \frac{1-\frac{\alpha}{2}}{(1-\alpha)^{2}}-b_{M}\right],
$$

which leads to the usual relation (Eq. (1)) if we take $\alpha=0$.

As shown by Eq. (33), the dimerization yield $\alpha$ depends on the equilibrium pressure $p$ which is taken to be $p=1 \mathrm{~atm}$, $K_{p}$ being usually given in $\mathrm{atm}^{-1}$ for this dimerization equilibrium. In order to solve Eq. (37), one thus has to know the $\alpha$ value corresponding to $p=1$ atm at the temperature $T$ of interest. Starting from the initial value $\alpha=0$, one iterates over first determining $K_{p}(T)$ from Eq. (37), which then provides a new 
$\alpha$ value by means of Eq. (33), until convergence. The influence of considering a nonzero $\alpha$ in Eq. (37) will be discussed in Sec. III C when we present the actual values of $K_{p}(T)$ obtained from the SVC $B(T)$ computed in Sec. II D. But it can already be realized that it will lead to an increase in the $K_{p}(T)$ constant with respect to assuming $\alpha=0$ as $1-\frac{\alpha}{2}>(1-\alpha)^{2}$.

\section{B. Excluded volume $b_{M}(T)$}

Progress has been made recently concerning the notion of excluded volume, in relation to the Generic van der Waals equation, which naturally gives rise to a statistical mechanical definition of a mean excluded volume (see, e.g., Ref. 68). We will use below a simplistic formulation as the corrective $b_{M}$ term appearing in Eq. (36) is defined as the intrinsic SVC of the monomer. For the simple case of the hard sphere potential, it corresponds to the well known expression $b_{0}=\frac{2}{3} \pi \sigma^{3}$, where $\sigma$ is the minimum approach distance. ${ }^{69}$ By analogy, for an arbitrary potential $V$ we define this intrinsic coefficient by the integral (similar to Eq. (31))

$$
b_{M}(T)=\frac{1}{128 \pi^{4}} \int \ldots \int \mathrm{H}\left[\mathrm{V}-\mathrm{E}_{\mathrm{thr}}\right] \mathrm{d} \mathbf{R} \mathrm{d} \boldsymbol{\Omega}^{\mathrm{A}} \mathrm{d} \boldsymbol{\Omega}^{\mathrm{B}},
$$

where $\mathrm{H}$ is the Heaviside function and $E_{t h r}$ is an energy threshold. It corresponds to the classically excluded volume above energy $E_{t h r}$, and gives the $\frac{2}{3} \pi \sigma^{3}$ factor for the hard sphere potential. We define $E_{t h r}=\frac{3}{2} k_{B} T$ which is the mean relative kinetic energy at temperature $T$, which renders $b_{M}$ temperature dependent.

A plot of $b_{M}(T)$ per $m o l$ is given in Fig. 2 in the range 200-600 K. After a sharp initial decrease, it coincides at room temperature with the $38.5 \mathrm{~cm}^{3} \mathrm{~mol}^{-1}$ value recommended by Hirschfelder and co-workers, ${ }^{37}$ and reaches the $31 \mathrm{~cm}^{3} \mathrm{~mol}^{-1}$ value currently used in the van der Waals equation. The notion of excluded volume for water vapor has recently been discussed by Tretyakov et al. ${ }^{70}$ They point out that "the excluded volume should be interpreted as the effective volume of intermolecular interaction due to the repulsive forces." These authors also conclude from their analysis that "it will first vary abruptly, probably exponentially, and then will decrease very slowly, remaining almost unchanged."

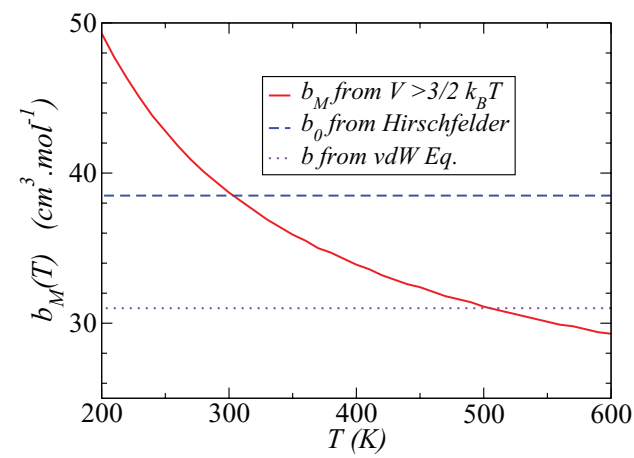

FIG. 2. Excluded volume $b_{M}(T)$ defined as the classically forbidden volume at temperature $T$ (see Eq. (38)).

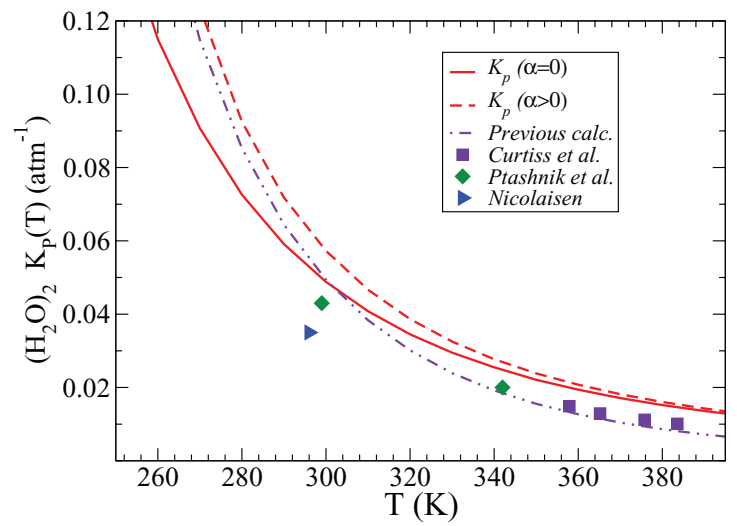

FIG. 3. Calculated equilibrium constant $K_{p}(T)$ in the range 250-400 K: (-) in the limit $\alpha=0,(--)$ considering the finite value of $\left.\alpha,{ }_{(} . ._{-}\right)$from our previous calculation ${ }^{30}$. Experimental data: (ם) results based on the thermal conductivity of steam experiments of Curtiss et al.; ${ }^{13}$ ( ) Ptashnik et al. ${ }^{15}$ high resolution pure water vapor absorption spectra using the Ma and Tipping continuum model; ( ) Nicolaisen ${ }^{17}$ IR absorption spectrum of water in $\mathrm{CCl}_{4}$.

\section{Equilibrium constant $K_{p}(T)$}

The equilibrium constant $K_{p}(T)$ has been determined from Eq. (37), using the SVC $B(T)$ obtained from Eq. (31) and the excluded volume $b_{M}(T)$ defined in Eq. (38). As already mentioned before, its values as determined from Eq. (37) depend on the dimerization yield $\alpha$ corresponding to an equilibrium pressure $p=1 \mathrm{~atm}$. We thus report in Fig. 3 the results obtained either from solving Eq. (37) $\left(K_{p}(\alpha>0)\right)$ or in the $\alpha \rightarrow 0$ limit $\left(K_{p}(\alpha=0)\right.$ ), in the range $260-400 \mathrm{~K}$, as well as our previous results. ${ }^{30}$ In Figure 3 are also represented the experimental results obtained by

(i) Curtiss et al., ${ }^{13}$ at high temperatures, based on the thermal conductivity of steam experiments;

(ii) Ptashnik et al., ${ }^{15}$ at temperatures $299 \mathrm{~K}$ and $342 \mathrm{~K}$, from high spectral resolution pure water vapor absorption spectra, corrected from the Ma and Tipping continuum model ${ }^{71}$ and using then water dimer intensities calculated by Schofield and Kjaergaard; ${ }^{72}$

(iii) Nicolaisen ${ }^{17}$ from IR absorption spectrum (4100-3200 $\mathrm{cm}^{-1}$ ) of water in $\mathrm{CCl}_{4}$ solutions at $296 \mathrm{~K}$, based on measurements of the atmospheric water absorption continuum $^{73}$ and calculations of the line strengths for the dimer. ${ }^{74}$

Let first compare the new results with our previous calculation. ${ }^{30}$ As was mentioned before, the empirical potential used previously was deeper $\left(D_{0} \simeq 1230 \mathrm{~cm}^{-1}\right)$ than the CCpol-8sf potential used in this work $\left(D_{0}=1108 \mathrm{~cm}^{-1}\right)$, which is in very good agreement with the experimental value $1105 \pm 10 \mathrm{~cm}^{-1}$ recently measured by Rocher-Casterline et al..$^{36}$ by velocity map imaging. From the influence of the dissociation energy $D_{0}$ on $K_{p}$ (Eq. (2)), one expects a global decrease in the new calculations, as shown at lower temperatures. On the other hand, resonances were not taken into account, in contrast to the method used in the present work. This should result in an increase of $K_{p}$ when these resonances become important, i.e., at higher temperatures. 
If we consider now the influence of the nonzero value of the dimerization yield $\alpha$ in Eq. (37), one can notice that it does play a significant role below ca. $320 \mathrm{~K}$. For example, this yield $\alpha$ varies from 0.17 to 0.04 in the range $260-320 \mathrm{~K}$.

The actual comparison to recent spectroscopic determinations of the constant is made difficult by their scarcity and the widely different experimental methods involved. One can however note a reasonable agreement with the estimations of Ptashnik et al. ${ }^{15}$ at two different temperatures.

\section{DISCUSSION}

We reported SVC calculations relying, for the first time, on a quantum implementation of the Takahashi-Imada approximation, to the difference of its usual classical statistical calculation through Monte Carlo sampling. This quantum formulation allowed us to retrieve the Path Integral Monte Carlo results obtained by Schenter $^{58}$ in the low temperature range 250-450 K for the model TIP4P potential. Using a water dimer formulation and an $a b$ initio flexible potential recently introduced, ${ }^{49}$ we first computed the SVC taking fully into account the monomers' flexibility. This new potential lead to a very good agreement with available experimental values. It was shown that flexibility of monomers has to be considered as it leads, e.g., to a $13 \%$ change at $300 \mathrm{~K}$ due to the asymptotic behavior of this coefficient at low temperatures. The SVC evaluation was made particularly efficient by use of a pseudospectral scheme based on Fourier and Gaussian quadratures, fully benefiting from parallelization.

We then extracted from these results the dimerization equilibrium constant $K_{p}(T)$ by means of the relation $K_{p}(T)$ $=-\left(B(T)-b_{M}\right) / R T$. We proposed a simplistic excluded volume $b_{M}$ definition, assimilated to the SVC of a pure water monomer vapor, and computed as the classically excluded volume for the mean relative kinetic energy $\frac{3}{2} k_{B} T$ at temperature $T$. It displays a slight dependence on temperature in accordance with the empirical formula used by Ben-Amotz and Herschbach, ${ }^{75} \sigma(T)=\sigma_{0}\left[1+\sqrt{T / T_{0}}\right]^{-\eta}$, to summarize the various effective diameters $\sigma(T)$ of molecules proposed in the literature. It allowed us to retrieve at room temperature the $38.5 \mathrm{~cm}^{3} \mathrm{~mol}^{-1}$ value recommended by Hirschfelder and coworkers, ${ }^{37}$ and reaches around $500 \mathrm{~K}$ the $31 \mathrm{~cm}^{3} \mathrm{~mol}^{-1}$ value currently used in the van der Waals equation. At room temperature or below, this excluded volume plays a marginal role in the equilibrium constant calculation as $-B(T)$ is much bigger. But its contribution changes from important (10\%) to essential $(100 \%)$ in the range $400-1000 \mathrm{~K}$. It should be noted that the higher the temperature, the more valid the classical approximation used to define $b_{M}(T)$.

It was mentioned before in Sec. III that metastable states do contribute to the SVC, and hence to the equilibrium constant according to the method used for its evaluation in this work. Two remarks then arise: (i) how do these metastable or resonant states contribute to these functions?; (ii) how the $K_{p}(T)$ values obtained in this way are related to the experimental ones obtained from absorption spectra? To answer the first point, one can quote Stogryn and Hirschfelder: ${ }^{14}$ "If the half-life for dissociation is greater than the average time between collisions, the resonances behave like bound states. But if the time between collisions is long compared to this half-life, they behave like free molecules." Their assertion can be understood as the SVC depends on the trace $\operatorname{Tr}\left[e^{-\beta \mathbf{H}_{A B}}-e^{-\beta \mathbf{H}_{A B}^{o}}\right]$. Sharp resonances are strongly localized above the interaction well, that is where $\mathbf{H}_{A B}$ and $\mathbf{H}_{A B}^{o}$ differ most, and henceforth their contribution to the trace will be more important. On the converse, broad resonances extend well beyond the interaction region where $\mathbf{H}_{A B}$ converges to $\mathbf{H}_{A B}^{o}$, resulting in a smaller contribution.

This discussion leads to the second point as it highlights the importance of the experiment timescale. As noted by Schenter et al., 48 "states above dissociation may correspond to collision complexes or resonances that are too short lived to contribute to any physically observable property of the dimer." Similarly, Vigasin ${ }^{76}$ pointed out the difference between "statistical" and "spectroscopical" definitions of short-lived pair states: "the use of various spectroscopic methods to probe molecular pairs may result in the identification of various fractions of short-lived states either with stable or with quasistable species." An accurate comparison to experiment should thus take into account its timescale and consider it the calculation of $K_{p}(T)$.

\section{ACKNOWLEDGMENTS}

This work was funded by the Agence Nationale de la Recherche Grant No. ANR-12-BS08-0010-01. N. Moiseyev, O. Atabek, and G. Jolicard are gratefully acknowledged for helpful discussions concerning resonant states, and $\mathrm{K}$. Szalewicz, M. Suhm, and M. Tretyakov for a critical reading of the manuscript.

${ }^{1}$ P. Varanasi, S. Chou, and S. S. Penner, J. Quant. Spectrosc. Radiat. Transfer 8, 1537 (1968)

${ }^{2}$ J. S. Daniel, S. Solomon, R. W. Sanders, R. W. Portman, D. C. Miller, and W. J. Madsen, J. Geophys. Res., [Atmos.] 104, 16785, doi:10.1029/1999JD900220 (1999).

${ }^{3}$ P. Chylek and D. J. Geldart, Geophys. Res. Lett. 24, 2015, doi:10.1029/97GL01949 (1997).

${ }^{4}$ G. T. Evans and V. Vaida, J. Chem. Phys. 113, 6652 (2000).

${ }^{5}$ V. Vaida, J. S. Daniel, H. G. Kjaergaard, L. M. Goss, and A. F. Tuck, Q. J. R. Meteorol. Soc. 127, 1627 (2001).

${ }^{6}$ V. Vaida, H. G. Kjaergaard, and K. J. Feierabend, Int. Rev. Phys. Chem. 22, 203 (2003).

${ }^{7}$ A. D. Devir, M. Neumann, S. G. Lipson, and U. P. Oppenheim, Opt. Eng. 33, 746 (1994).

${ }^{8}$ J. S. Daniel, S. Solomon, H. G. Kjaergaard, and D. P. Schofield, Geophys. Res. Lett. 31, L06118, doi:10.1029/2003GL018914 (2004).

${ }^{9}$ H. R. Pruppacher and J. D. Klett, Microphysics of Clouds and Precipitation (Reidel, Norwell, MA, 1978).

${ }^{10}$ G. K. Schenter, S. M. Kathmann, and B. C. Garett, Phys. Rev. Lett. 82, 3484 (1999).

${ }^{11}$ T. Loerting and K. R. Liedl, Proc. Natl. Acad. Sci. U.S.A. 97, 8874 (2000).

${ }^{12}$ S. Aloiso, J. S. Francisco, and R. R. Friedl, J. Phys. Chem. A 104, 6597 (2000).

${ }^{13}$ L. A. Curtiss, D. J. Frurip, and M. Blander, J. Chem. Phys. 71, 2703 (1979).

${ }^{14}$ D. E. Stogryn and J. O. Hirschfelder, J. Chem. Phys. 31, 1531 (1959).

${ }^{15}$ I. V. Ptashnik, K. M. Smith, K. P. Shine, and D. A. Newnham, Q. J. R. Meteorol. Soc. 130, 2391 (2004).

${ }^{16}$ I. V. Ptashnik, J. Quant. Spectrosc. Radiat. Transfer 109, 831 (2008).

${ }^{17}$ F. M. Nicolaisen, J. Quant. Spectrosc. Radiat. Transfer 110, 2060 (2009).

${ }^{18}$ I. V. Ptashnik, K. P. Shine, and A. A. Vigasin, J. Quant. Spectrosc. Radiat. Transfer 112, 1286 (2011)

${ }^{19}$ A. J. L. Schillings, S. M. Ball, M. J. Barber, J. Tennyson, and R. L. Jones, Atmos. Chem. Phys. 11, 4273 (2011). 
${ }^{20}$ M. Y. Tretyakov, E. A. Serov, M. A. Koshelev, V. V. Parshin, and A. F. Krupnov, Phys. Rev. Lett. 110, 093001 (2013).

${ }^{21}$ D. A. McQuarrie, Statistical Mechanics (Harper and Row, New York, 1976).

${ }^{22}$ H. G. Kjaergaard, T. W. Robinson, D. L. Howard, J. S. Daniel, J. E. Headrick, and V. Vaida, J. Phys. Chem. A 107, 10680 (2003).

${ }^{23}$ K. H. Lemke and T. M. Seward, J. Geophys. Res., [Atmos.] 113, D19304, doi:10.1029/2007JD009148 (2008).

${ }^{24}$ Z. Slanina and J. F. Crifo, Int. J. Thermophys. 13, 465 (1992).

${ }^{25}$ N. R. Zhang and D. D. Shillady, J. Chem. Phys. 100, 5230 (1994).

${ }^{26}$ C. Munoz-Caroz and A. Nino, J. Phys. Chem. A 101, 4128 (1997).

${ }^{27}$ F. Kollipost, R. Larsen, A. V. Domanskaya, M. Nörenberg, and M. A. Suhm, J. Chem. Phys. 136, 151101 (2012).

${ }^{28}$ N. Goldman, R. S. Fellers, C. Leforestier, and R. J. Saykally, J. Phys. Chem. A 105, 515 (2001).

${ }^{29}$ N. C. Leforestier and R. J. Saykally, J. Phys. Chem. A 108, 787 (2004).

${ }^{30}$ Y. Scribano, N. Goldman, R. J. Saykally, and C. Leforestier, J. Phys. Chem. A 110, 5411 (2006).

${ }^{31}$ C. Leforestier, F. Gatti, R. S. Fellers, and R. J. Saykally, J. Chem. Phys. 117, 8710 (2002).

${ }^{32}$ J. M. Bowman, Adv. Chem. Phys. 61, 115 (1985).

${ }^{33}$ S. L. Mielke, G. C. Lynch, D. G. Truhlar, and D. W. Schwenke, Chem. Phys. Lett. 216, 441 (1993).

${ }^{34}$ A. A. Vigasin, Mol. Phys. 108, 2309 (2010).

${ }^{35}$ T. A. Odintsova and M. Y. Tretyakov, J. Quant. Spectrosc. Radiat. Transfer 120, 134 (2013).

${ }^{36}$ B. E. Rocher-Casterline, L. C. Ch'ng, A. K. Mollner, and H. Reisler, J. Chem. Phys. 134, 211101 (2011).

${ }^{37}$ J. O. Hirschfelder, F. T. CcClure, and I. F. Weeks, J. Chem. Phys. 10, 201 (1942).

${ }^{38}$ D. E. Stogryn and J. O. Hirschfelder, J. Chem. Phys. 31, 1545 (1959).

${ }^{39}$ F. T. Smith, J. Chem. Phys. 38, 1304 (1963).

${ }^{40}$ F. T. Smith, Phys. Rev. 131, 2803 (1963).

${ }^{41}$ R. T Pack, E. A. Butcher, and G. A. Parker, J. Chem. Phys. 102, 5998 (1995).

${ }^{42}$ J. C. Rainwater, J. Chem. Phys. 81, 495 (1984).

${ }^{43}$ A. A. Vigasin, Infrared Phys. 32, 461 (1991).

${ }^{44}$ S. Yu. Epifanov and A. A. Vigasin, Mol. Phys. 90, 101 (1997).

${ }^{45}$ A. A. Vigasin, in Molecular Complexes in Earth's, Planetary, Cometary, and Interstellar Atmospheres, edited by A.A.Vigasin and Z.Slanina (World Scientific Publishing, Singapore, 1998).

${ }^{46}$ A. A. Vigasin, J. Quant. Spectrosc. Radiat. Transfer 64, 25 (2000).

${ }^{47}$ G. K. Schenter, J. Chem. Phys. 108, 6222 (1998).

${ }^{48}$ G. K. Schenter, S. M. Kathmann, and B. C. Garett, J. Phys. Chem. A 106, 1557 (2002)
${ }^{49}$ C. Leforestier, K. Szalewicz, and A. van der Avoird, J. Chem. Phys. 137, 014305 (2012).

${ }^{50}$ G. Brocks, A. der Avoird, B. T. Sutcliffe, and J. Tennyson, Mol. Phys. 50, 1025 (1983).

${ }^{51}$ T. R. Dyke, J. Chem. Phys. 66, 492 (1977).

${ }^{52}$ C. Leforestier, L. B. Braly, K. Liu, M. J. Elrod, and R. J. Saykally, J. Chem. Phys. 106, 8527 (1997).

${ }^{53}$ G. R. Low and H. G. Kjaergaard, J. Chem. Phys. 110, 9104 (1999).

${ }^{54}$ Y. Wang and J. M. Bowman, Chem. Phys. Lett. 491, 1 (2010).

${ }^{55}$ P. E. S. Wormer, J. Chem. Phys. 122, 184301 (2005).

${ }^{56}$ R. T Pack, J. Chem. Phys. 78, 7217 (1983).

${ }^{57}$ M. Takahashi and I. Imada, J. Phys. Soc. Jpn. 53, 3765 (1984).

${ }^{58}$ G. K. Schenter, J. Chem. Phys. 117, 6573 (2002).

${ }^{59}$ E. M. Mas, R. Bukowski, K. Szalewicz, G. C. Gronenboom, P. E. S. Wormer, and A. der Avoird, J. Chem. Phys. 113, 6687 (2000).

${ }^{60}$ W. L. Jorgensen and J. D. Madura, J. Am. Chem. Soc. 105, 1407 (1983).

${ }^{61}$ W. Cencek, K. Szalewicz, C. Leforestier, R. van Harrevelt, and A. van der Avoird, Phys. Chem. Chem. Phys. 10, 4716 (2008).

${ }^{62}$ N. S. Osborne, H. F. Stimson, and D. C. Ginnings, J. Res. Natl. Bur. Stand. 18, 389 (1937)

${ }^{63}$ P. T. Eubank, L. L. Joffrion, M. R. Patel, and W. Warowny, J. Chem. Thermodyn. 20, 1009 (1988).

${ }^{64}$ G. S. Kell, G. E. McLaurin, and E. Whalley, Proc. R. Soc. London, Ser. A 425, 49 (1989)

${ }^{65}$ A. H. Harvey and E. W. Lemmon, J. Phys. Chem. Ref. Data 33, 369 (2004).

${ }^{66}$ A. G. Donchev, N. G. Galkin, and V. I. Tarasov, Phys. Rev. Lett. 97, 220401:1 (2006).

${ }^{67}$ J. D. Lambert, G. H. A. Roberts, J. S. Rowlinson, and V. J. Wilkinson, Proc. R. Soc. London, Ser. A 196, 113 (1949).

${ }^{68}$ R. Laghaei, A. E. Nasrabad, and B. C. Eu, J. Chem. Phys. 124, 154502 (2006).

${ }^{69}$ O. K. Rice, Statistical Mechanics, Thermodynamics and Kinetics (Freeman, San Francisco, 1967).

${ }^{70}$ M. Y. Tretyakov, E. A. Serov, and T. A. Odintsova, Radiophys. Quantum Electron. 54, 700 (2012).

${ }^{71}$ Q. Ma and R. H. Tipping, J. Chem. Phys. 116, 4102 (2002).

${ }^{72}$ D. P. Schofield, H. G. Kjaergaard, and D. P. Schofield, Phys. Chem. Chem. Phys. 5, 3100 (2003).

${ }^{73}$ D. J. Paynter, I. V. Ptashnik, K. P. Shine, and K. M. Smith, Geophys. Res. Lett. 34, L12808, doi:10.1029/2007GL029259 (2007).

${ }^{74}$ H. G. Kjaergaard, A. L. Garden, G. M. Chaban, D. A. Matthews, and J. F. Stanton, J. Phys. Chem. A 112, 4324 (2008).

${ }^{75}$ D. Ben-Amotz and D. R. Herschbach, J. Phys. Chem. 94, 1038 (1990).

${ }^{76}$ A. A. Vigasin, in Weakly Interacting Molecules Pairs: Bimolecular Absorption in Atmospheric Gases, edited by C.Camy-Peyret and A.A.Vigasin (Kluwer Academic, 2003). 